\title{
Magnetic Resonance Relaxometry at Low and Ultra low Fields
}

\author{
P. Volegov, M. Flynn, R. Kraus, P. Magnelind, A. Matlashov, P. Nath, T. Owens, H. Sandin, I. \\ Savukov, L. Schultz, A. Urbaitis, V. Zotev, and M. Espy \\ Applied Modern Physics, Los Alamos National Laboratory, Los Alamos, NM USA
}

\section{Abstract}

Nuclear magnetic resonance (NMR) and magnetic resonance imaging (MRI) are ubiquitous tools in science and medicine. NMR provides powerful probes of local and macromolecular chemical structure and dynamics. Recently it has become possible and practical to perform MR at very low fields (from $1 \mu \mathrm{T}$ to $1 \mathrm{mT}$ ), the so-called ultra-low field (ULF) regime. Pulsed pre-polarizing fields greatly enhance the signal strength and allow flexibility in signal acquisition sequences. Improvements in SQUID sensor technology allow ultra-sensitive detection in a pulsed field environment.

In this regime the proton Larmor frequencies $(1 \mathrm{~Hz}-100 \mathrm{kHz}$ ) of ULF MR overlap (on a time scale of $10 \mu$ s to $100 \mathrm{~ms}$ ) with "slow" molecular dynamic processes such as diffusion, intramolecular motion, chemical reactions, and biological processes such as protein folding, catalysis and ligand binding. The frequency dependence of relaxation at ultra-low fields may provide a probe for biomolecular dynamics on the millisecond timescale (protein folding and aggregation, conformational motions of enzymes, binding and structural fluctuations of coupled domains in allosteric mechanisms) relevant to host-pathogen interactions, biofuels, and biomediation. Also this resonance-enhanced coupling at ULF can greatly enhance contrast in medical applications of ULF-MRI resulting in better diagnostic techniques.

We have developed a number of instruments and techniques to study relaxation vs. frequency at the ULF regime. Details of the techniques and results are presented.

Ultra-low field methods are already being applied at LANL in brain imaging, and detection of liquid explosives at airports. However, the potential power of ultra-low field MR remains to be fully exploited.

\section{Keywords}

ultra-low field; nuclear magnetic resonance; relaxation

\section{Introduction}

Nuclear magnetic resonance (NMR) and magnetic resonance imaging (MRI) are ubiquitous tools in science and medicine. NMR provides powerful probes of local and macromolecular chemical structure and dynamics. In spite of that the main trend in NMR/MRI industry favors instruments with higher and higher magnetic fields - mainly due to higher signal-tonoise ratio at higher fields - in recent years several research groups all over the world have started or continued work in the area of NMR at low and ultra-low fields. Due to utilization of magnetically shielded enclosures, pre-polarizing magnetic field and improvements in SQUID sensor technology allowing ultra-sensitive detection in a pulsed field environment, it has become possible and practical to perform MR at very low fields (from $1 \mu \mathrm{T}$ to $1 \mathrm{mT}$ ), the so-called ultra-low field (ULF) regime. 
The ULF regime has a number of unique features which can be advantageously exploited in a number of applications. First, due to low measurement field strength, it is much easier to create a field with required absolute uniformity. For example, if we need to ensure that frequency spread over the sample is less than $1 \mathrm{~Hz}$ (protons) for a $1 \mathrm{~T}$ NMR system, we need to shim the coils to $0.02 \mathrm{ppm}$ accuracy, while for the $100 \mu \mathrm{T}$ system the shimming requirement is much less stringent $\sim 200 \mathrm{ppm}$. This allows building rather simple and inexpensive magnetic systems with open access and convenience of measurement of large samples. Second, due to low Larmor frequency ( $4.25 \mathrm{kHz}$ at $100 \mu \mathrm{T}$ for protons), it is possible to make NMR/MRI measurements of the samples with metal inclusions (implants, bullets, etc) and even completely enclosed by a metal container, such as a soda can. Third, and this is probably the most important and promising feature of the ULF regime, the Larmor frequency in this mode overlap (on a time scale of $10 \mu$ s to $100 \mathrm{~ms}$ ) with the rate of "slow" molecular dynamic processes such as diffusion, intra-molecular motion, chemical reactions, and biological processes such as protein folding, catalysis and ligand binding. Although conventional NMR techniques can probe some of these processes, resonanceenhanced coupling at ULF can greatly enhance sensitivity of the relaxation measurements, thus yielding new information about these processes, and lead to the enhanced contrast.

Our team at Los Alamos has developed several applications of ULF MR including imaging of the human brain at $46 \mu \mathrm{T}$ in combination with magnetoencephalography [1], classification of materials via relaxation properties measured at ULF relevant to numerous non-invasive inspection applications, and determination of uranium enrichment fraction via relaxation and/or J-coupling to address nuclear safeguards [2], [3]. Below we discuss the motivation, methods, and recent results in imaging and relaxometry of materials using the ULF MR approach.

\section{METHODS AND INSTRUMENTS}

The most widely used technique to measure NMR relaxation at low fields consists of polarizing the sample in a large field $B_{p}$, then, switching off $B_{p}$ in a time short compared to $T_{1}$ and observing the free induction decay (FID) of the magnetization of the sample at a low field $B_{m}$. If the polarization field is switched off adiabatically $d B / d t \ll \gamma B^{2}$, where $\gamma$ is magnetogyric ratio) the bulk magnetization becomes aligned along the measurement field $B_{m}$ and it is then necessary to apply a $\pi / 2$ pulse to observe the precession of the

magnetization. Alternatively, if $B_{p}$ is switched off non-adiabatically $\left(d B_{p} / d t \geq \gamma B_{m}^{2}\right.$ ) then the magnetization remains aligned along $B_{p}$ and, if $B_{m}$ is not parallel to $B_{p}$, the precession of the bulk magnetization is directly observable [4].

In our experiments the typical range of the polarization field is from 10 to $50 \mathrm{mT}$, while the range of the measurement field is from 5 to $200 \mu \mathrm{T}$. This protocol intrinsically allows measuring two very different relaxation parameters: 1 ) longitudinal relaxation $\left(T_{1}\right)$ at relatively high field (Larmor frequency in $\mathrm{MHz}$ range for protons) by varying the polarization time, and 2) transverse relaxation $\left(T_{2}\right)$ at low field ( $\mathrm{kHz}$ range) by measuring decay of the signal with time. Such combination of the relaxation parameters proved to be very useful for characterizing materials. This can be qualitatively illustrated using the simplest model for NMR relaxation - an ensemble of spins which do not couple to one another but which couple to an external fluctuation field (Redfield theory, see for example [5]). According to this model the dependence of the relaxation times versus Larmor frequency $\omega_{L}$ can be expressed in terms of two microscopic quantities - an ensemble average of the local magnetic field $\overline{B_{l o c}^{2}}$ and correlation time $\tau_{0}$ : 


$$
\begin{gathered}
\frac{1}{T_{1}}=\frac{2}{3} \gamma^{2} \overline{B_{l o c}^{2}} \frac{\tau_{0}}{1+\left(\omega_{L} \tau_{0}\right)^{2}} \\
\frac{1}{T_{2}}=\frac{1}{3} \gamma^{2} \overline{B_{l o c}^{2}}\left[\tau_{0}+\frac{\tau_{0}}{1+\left(\omega_{L} \tau_{0}\right)^{2}}\right]
\end{gathered}
$$

As the Larmor frequency during the polarization and the measurement intervals differs by 3 order of magnitude, $T_{1}$ and $T_{2}$ measurements in this case reflect different properties of the relaxation process or even different relaxation mechanisms.

In contrast to conventional NMR/MRI, relative homogeneity of the measurement field is not crucial in ULF MRI, because microtesla-range magnetic fields of even modest relative homogeneity are highly homogeneous on the absolute scale. In this case the signal attenuation by effects of the dephasing and diffusion due to field non-uniformity over the sample volume is negligible and this makes possible to measure transverse relaxation directly from the free inductance decay signal even for relatively large samples $(\sim 100 \mathrm{cc})$, avoiding use of spin-echo sequences, such as CPMG. First, this approach significantly simplifies the experimental apparatus - no spin flipping pulses, coils, synchronization, etc. Second, and this is probably more important, this approach also provides more data points (more dense sampling) of the transverse relaxation decay. It should be noted that the transverse relaxation data is typically a sum of exponentials associated to the different components of the sample (such as for example as water compartmentalization in biological tissue). Resolving the such data into individual components is very important for characterizing the sample. However, this problem is ill-conditioned and the results strongly depend on the quality of the initial relaxation curve measurement making the solutions difficult to interpret and validate [6]. In this context dense sampling of a relaxation curve improves the ability to resolve such data and extract the underlying characteristic relaxation times. At last, it should be noted that direct measurement of the relaxation curve preserves the information about local non-uniformity of the magnetic fields in the sample, such for example as the one associated with paramagnetic impurities or contrast agents, while spinecho sequences mostly cancel these effects.

The approach to measure $T_{1}$ at high field and measure $T_{2}$ at low field, can be combined with a MRI sequence to obtain spatial maps of the relaxation parameters. An example of such a sequence - 3D Fourier multiple gradient echo imaging - is shown in the figure 1. The sequence consists of the pre-polarization, phase encoding, and several echo intervals created by inverting both the measurement field and the readout gradient. The images are obtained for the each echo, and for several different polarization times. The spin density map $\rho(\mathbf{r})$ and the relaxation maps $T_{1}(\mathbf{r}), T_{2}^{*}(\mathbf{r})$ are obtained by fitting the parameters of the following model (see for example [7]):

$$
I_{i j}(\mathbf{r}) \propto \rho(\mathbf{r}) \cdot\left(1-e^{-T_{p}^{(j)} / T_{1}(\mathbf{r})}\right) \cdot e^{-T_{e}^{(i)} / T_{2}^{*}(\mathbf{r})}
$$

where $\left\{T_{p}^{(j)}\right\}_{1}^{m}$ are the polarization times, $\left\{T_{e}^{(i)}\right\}_{1}^{n}$ are the echo times, and $I_{i j}(\mathbf{r})$ is the image obtained for $i$-th echo and $j$-th polarization time. To compensate for the effects of any static residual fields, a uniform water phantom is used to calibrate the system. At last, one should mention here that the ratio of the MRI signal bandwidth, defined by the gradient strength and the size of the sample, to the Larmor frequency is typically larger in the ULF MRI regime comparing to its high field counterpart. This dictates that effects of concomitant gradients should be taken into account [8]. 
Rapidly switching off $B_{p}$ inevitably results in the transient field which arises from the currents proportional to $d B / d t$ induced in any conductive materials (e.g. other components of the experimental apparatus and surrounding equipment). While the transient fields are significantly smaller than $B_{m}(\sim 1-10 \mu \mathrm{T})$, hence they do not significantly alter the evolution of the spins, they are much larger than the typical signal from bulk magnetization of the sample $(\sim 1 . .10 \mathrm{pT})$ thus impeding or even preventing measurement of the NMR signal and the associated NMR relaxation parameters. One approach to correct for the transient signals is to approximate the transients with a sum of exponents (typically 2-3) and subtract them in post processing. We have previously used this approach with good success. However this approach is rather limited to the extent it could cancel the transients, especially for shorter ( $10 \mathrm{~ms}$ ) periods. We designed a new technique to cancel transient-induced signals by acquiring interleaved NMR measurements at two different measurement fields (i.e. two different $\omega_{\mathrm{L}}$ ) and differencing these two signals. This method could cancel the transient signal by 3 orders of magnitude [9].

Over the last few years we developed an number of ULF NMR/MRI instruments ranging from a one channel NMR system designed to measure FID to multi-channel (7-channel) MRI system designed to image relaxation properties of multiple objects within one sample.

The detector of choice is a SQUID (a Superconducting QUantum Interference Device) [10]. This is due to the following two factors. First, the amplitude of the NMR signal at ULF is very low (for example a $1 \mathrm{cc}$ sample of water polarized by $20 \mathrm{mT}$ field generates $\sim 2.3 \mathrm{pT}$ field at the distance $3 \mathrm{~cm}$ from the sample), and, second, the Larmor frequency is also low, in the $\mathrm{kHz}$ range. The combination of these two factors makes it very difficult to detect the signal using a Faraday coil, but given the low intrinsic noise of the SQUID coupled with a untuned superconducting flux transformer, it is possible to achieve an acceptable signal-tonoise ratio [11]. The main challenge to use a SQUID detector in ULF NMR/MRI applications is to ensure that SQUID is in a working state after fast ramping down the polarization field. To protect the SQUID from the switching transients we use a fast cryogenic switch [12], which decouples the SQUID from the pick-up loops during the ramp down time, and then reconnects it within, typically, $5 \mu \mathrm{s}$. We also found it is very important to carefully isolate the SQUID chip itself from the ambient magnetic fields by a superconducting shield.

Another important and complex element of the systems is the pre-polarization magnet: a coil and power supply. Given a finite power supply, the design goal to generate as large polarization field as possible in the sample volume dictates using coils with relatively large number of turns ( 1000) placed in the immediate vicinity of the sample. This presents several problems. First, the inductance of the polarization coils - typically for our instruments it is in the range from $25 \mathrm{mH}$ to $500 \mathrm{mH}$ - determines how fast the polarization field could be ramped down: $\Delta t=L I / U$, where $L$ is the coil inductance, $I$ is the polarization current, $U$ is the voltage across the switching device. To address this problem we designed a current switching device based on optoMOS solid state relays [13]. The device is capable of switching on/off currents up to $80 \mathrm{~A}$. The voltage across the switch is up to $\sim 2.4 \mathrm{kV}$, which allows us to ramp down the polarization field in the time from 0.5 to $20 \mathrm{~ms}$, depending on the inductance of the coils and the current. Second, the polarization coil effectively couples noise from the power supply to the SQUID, so we employ mechanical relays in addition to the solid state relays to completely decouple the polarization coils from the external circuits during the measurement. Third, the large amount of the conducting material near the SQUID pick-up loops is bound to generate strong noise due to Johnson currents. To overcome this effect we are using multi-stranded litz wire. Last, but not least, it should be noted that the coils generate significant amount of heat during the polarization period which should be dissipated without overheating the coils and the sample. Typically for our instruments it 
ranges from 0.5 to $4 \mathrm{~kW}$. To remove the generated heat from the coils and sample volume we are using different cooling agents: air (convection and forced), liquid nitrogen (evaporation) [13], a fluorine-based industrial coolant (flow) [14].

\section{APPLICATIONS AND RESULTS}

Using the methods and instruments described in the previous section we have developed several applications of ULF MR designed for classification of materials via relaxation properties measured at ULF relevant to numerous non-invasive inspection applications.

Uranium hexafluoride $\left(\mathrm{UF}_{6}\right)$ is used in the enrichment process of uranium. It is a solid at room temperature and atmospheric pressure and there exists a triple point at $64{ }^{\circ} \mathrm{C}$. At temperatures above the triple point the $\mathrm{UF}_{6}$ consists of a mixture of liquid and vapor. A nondestructive ULF-NMR enrichment verification system could serve an important role in nonproliferation surveillance. We have developed an instrument to measure relaxation parameters of $\mathrm{UF}_{6}$ in liquid phase and (possibly in gas phase) at ULF regime. As the transverse relaxation time of $\mathrm{UF}_{6}$ is expected to be short (on the order of $10 \mathrm{~ms}$ for liquid and $1 \mathrm{~ms}$ for vapor) we employed the two frequency approach, described in the previous section, to minimize effects of the transients. FIDs have been recorded from a heated $\mathrm{UF}_{6}$ sample at a mean temperature of $80^{\circ} \mathrm{C}$. The results are shown in the figure 2 . The fluorine peaks have been detected and match the frequencies of a reference liquid fluorine sample (perfluorodecalin; $\mathrm{C}_{10} \mathrm{~F}_{18}$ ). The two-frequency approach was very effective in removing the transients and reducing the noise about 100 times. The FIDs have been analyzed using bootstrapping to find the corresponding spin-spin relaxation time $T_{2}$, a lognormal fit has been used to retrieve a mean of $24 \mathrm{~ms}$ and a variance of $13 \mathrm{~ms}$.

To address the issue of measuring relaxation properties of several objects in an arbitrary configuration, we developed an ULF imaging relaxometer - MagViz [14]. The relaxometer is being applied to a homeland security application inspecting liquids in a check-point, and determining the presence of hazardous material. The instrument has also been used to distinguish liquids, such as wine, for quality. Fig. 3 presents results from the relaxometer. The pseudo-color image reflects relaxation properties of the objects: the intensity (the value component in hue-saturation-value scheme) is proportional to the spin-density, while the hue and saturation are functions of $T_{1}$ and $T_{2}$. The tests were performed with benign items from streams of commerce, and a threat material, hydrogen peroxide. The 2D analysis integrated over the depth of the bowl which was $\sim 3$ inches. The 3D analysis is shown with three slices. Circles indicate correctly identified objects found to contain threat material. In the upper images a foil-lined juice box was used, and the material inside still correctly identified. The unit was also used to analyze the difference between several different types of wine and vinegar.

We applied the ULF MR relaxation imaging approach to medical applications including imaging relaxation properties of the human brain at $46 \mu \mathrm{T}$. We obtained following $\mathrm{T} 1$ values at $\mathrm{Br}=46 \mu \mathrm{T}$ relaxation field: $103 \pm 5 \mathrm{~ms}$ for gray matter, $75 \pm 2 \mathrm{~ms}$ for white matter, $344 \pm 9 \mathrm{~ms}$ for CSF, and $124 \pm 7 \mathrm{~ms}$ for scalp, and the following T2 values at the same field: $106 \pm 11 \mathrm{~ms}$ for gray matter, $79 \pm 11 \mathrm{~ms}$ for white matter, $355 \pm 15 \mathrm{~ms}$ for CSF, and $120 \pm 7 \mathrm{~ms}$ for scalp. The measurements confirm that at microtesla fields $T_{1}$-weighted contrast is similar to $T_{2}$ contrast for human brain tissues. This leads to the conclusion that the main advantage of ULF MRI is not the imaging contrast established at microtesla fields, but the unique opportunity to explore and utilize in vivo $T_{1}$-weighted contrast as a function of the relaxation field in a wide range of magnetic fields (from zero to $0.1 \mathrm{~T}$ and higher) [15]. 
One of the most interesting and important problems in neuroscience is to measure brain function. Two major techniques are widely used: a) magneto-encephalography (MEG), and b) functional MRI (fMRI). Each of these two techniques has its own advantages and drawbacks. As we have shown previously MEG can be combined with ULF MRI. We would like to evaluate plausibility of using fMRI in the ULF regime. fMRI measures changes in the relaxation properties of spin population in blood produced by changes in blood oxygenation during neural activity. The main mechanism which changes the relaxation rate is a change in susceptibility. This effect is dependent on the strength of the measurement field and so would be unlikely to be present at ULF. However, it is possible that there are other relaxation mechanisms present at ULF that are dependent on blood oxygenation. To answer that question, we measured the changes in $T_{1}$ and $T_{2}$ in blood before and after deoxygenation at ULF. Deoxygenation was performed by evacuating the blood to $540 \mathrm{~mm} \mathrm{Hg}$ for two hours. Measurements of $T_{1}$ at $18.8 \mathrm{mT}$ and $T_{2}$ at $46 \mu \mathrm{T}$ found $T_{1}$ of $351 \pm 29 \mathrm{~ms}$ and $T_{2}$ of $194 \pm 9 \mathrm{~ms}$ for the oxygenated blood and deoxygenated blood had a $T_{1}$ of $335 \pm 82 \mathrm{~ms}$ and $T_{2}$ of $194 \pm 9 \mathrm{~ms}$. Overall, we found no statistically significant difference in $T_{1}$ or $T_{2}$ values when the level of oxygenation was changed. However, it may still be possible to perform fMRI in the ULF regime using other approaches based on changes in blood volume or flow.

The intrinsic MRI tissue contrast at ultra-low elds (ULF) has been shown to be higher than at high-fields [1]. However, further improvement of the contrast would be beneficial. To evaluate the performance of contrast agents at ULF we measured relaxivity of several contrast agents at different fields. We have chosen superparamagnetic iron oxide particles (SPIOs) for this study. Feridex ${ }^{\circledR}[16]$ is an FDA approved SPIO contrast agent. We have also studied different nanoparticles [17]. Some of the results are shown in the figure 4 . The $R_{1}$ relaxivities were measured at the pre-polarization field $(50 \mathrm{mT})$. With a different scheme, $R_{1}$ measurement could be performed at the measurement field. However, we observed no change in $R_{2}$ when reducing the polarization field from $50 \mathrm{mT}$ to $25 \mathrm{mT}$. We found no consistent evidence of dispersion over the frequency range $0.8-7.3 \mathrm{kHz}$ although there might be some shift at the lower part of the range. The main conclusion are a) iron oxide nano-particles work as contrast agents at ultra-low fields, b) the $T_{1}$ contrast enhancement for Feridex is higher at low field $(50 \mathrm{mT})$ than at typical MRI fields $(\geq 1.5 \mathrm{~T})$.

\section{CONCLUSIONS}

Using ULR MR relaxometry we have made progress in numerous applications ranging from brain imaging to homeland security to industry. These applications illustrate the promise of the ULF relaxomentry approach to magnetic resonance as a scientific and industrial tool. However, the real potential of the method remains to be determined. While some relaxation mechanisms are already understood and experimentally verified at high field, ultra-low-field relaxation behavior has not been rigorously studied experimentally or theoretically. ULF MR provides a tool to study relaxation vs. frequency at ultra-low frequencies, to characterize relaxation behavior and hence the underlying molecular dynamics. One of the most promising applications of ULF MR is the study of the "slow" dynamic processes. Enhanced relaxation contrast in biological tissue, a unique benefit of imaging at ULF, is presumably the result of these complicated motions. Yet the theory of MR relaxation, which provides a window into these dynamics, has not been developed and validated below $10 \mathrm{kHz}$. The frequency dependence of relaxation at ULF may provide an exciting probe for molecular dynamics on the millisecond timescale (i.e. protein folding and aggregation, conformational motions of enzymes, binding and structural fluctuations of coupled domains in allosteric mechanisms) relevant to a host of applications yet to be discovered. 


\section{Acknowledgments}

This work was done with support from the U.S. Department of Homeland Security, Department of Energy Office of Biological and Environmental Research, the National Institutes of Health R01 EB006456, and support from Los Alamos National Laboratory LDRD.

\section{References}

1. Zotev VS, Matlashov AN, Volegov PL, Savukov IM, Espy MA, Mosher JC, Gomez JJ, Kraus RH Jr. Microtesla MRI of the human brain combined with MEG. Journal of Magnetic Resonance. 2005; 175:103-113. [PubMed: 15869890]

2. Volegov PL, Matlashov AN, Kraus RH Jr. Ultra-low field NMR measurements of liquids and gases with short relaxation times. Journal of Magnetic Resonance. 2006; 183:134-141. [PubMed: 16945561]

3. Magnelind P, Matlashov A, Volegov P, Espy M. Ultra-Low Field NMR of UF6 for 235U Detection and Characterization. IEEE Transactions on Applied Superconductivity. June; 2009 19(3):816-818.

4. Abragam, A. Principles of Nuclear Magnetism. Oxford University Press; 1961.

5. Slichter, CP. Principles of Magnetic Resonance. Springer; 1990. p. 206-212.

6. Andrade L, Micklander E, Farhat I, Bro R, Engelsen SB. DOUBLESLICING: A non-iterative single profile multi-exponential curve resolution procedure Application to time-domain NMR transverse relaxation data. Journal of Magnetic Resonance. 2007; 189:286-292. [PubMed: 17950014]

7. Callaghan, PT. Principles of Nuclear Magnetic Resonance Microscopy. Oxford; 2004.

8. Volegov PL, Mosher JC, Espy MA, Kraus RH Jr. On concomitant gradients in low-field MRI. Journal of Magnetic Resonance. 2005; 175:103-113. [PubMed: 15869890]

9. Volegov PL, Matlashov AN, Kraus RH Jr. Ultra-low field NMR measurements of liquids and gases with short relaxation times. Journal of Magnetic Resonance. 2006; 183:134-141. [PubMed: 16945561]

10. Clarke, John; Braginski, Alex I. The SQUID Handbook. Vol. I. Wiley-VCH Verlag GmbH \& Co. $\mathrm{KGaA} ; 2004$.

11. Clarke, John; Braginski, Alex I. The SQUID Handbook. Vol. II. Wiley-VCH Verlag GmbH \& Co. $\mathrm{KGaA} ; 2004$.

12. "SQUID sensor Model CE2 blue and cryoswitch Model SW1 from Supracon AG," Jena, Germany [Online]. Available: www.supracon.com

13. Zotev V, Matlashov A, Volegov P, Urbaitis A, Espy M, Kraus R Jr. SQUID-based instrumentation for ultralow-field MRI. Supercond Sci Technol. 2007; 20:S367-S373.

14. Espy M, Flynn M, Gomez J, Hanson C, Kraus R, Magnelind P, Maskaly K, Matlashov A, Newman S, Owens T, Peters M, Sandin H, Savukov I, Schultz L, Urbaitis A, Volegov P, Zotev V. Ultra-low Field MRI for the Detection of Liquid Explosives Using SQUIDs. IEEE Transactions on Applied Superconductivity. June; 2009 19(3):835-838.

15. Zotev VS, Matlashov AN, Savukov IM, Owens T, Volegov PL, Gomez JJ, Espy MA. SQUIDBased Microtesla MRI for In Vivo Relaxometry of the Human Brain. IEEE Transactions on Applied Superconductivity. June; 2009 19(3):823-826.

16. AMAG Pharmaceuticals, Inc; 100 Hayden Ave, Lexington, MA 02421, USA: http://www.amagpharma.com

17. Ocean NanoTech, LLC; 2143 Worth Ln., Springdale, AR 72764, USA: http://www.oceannanotech.com 


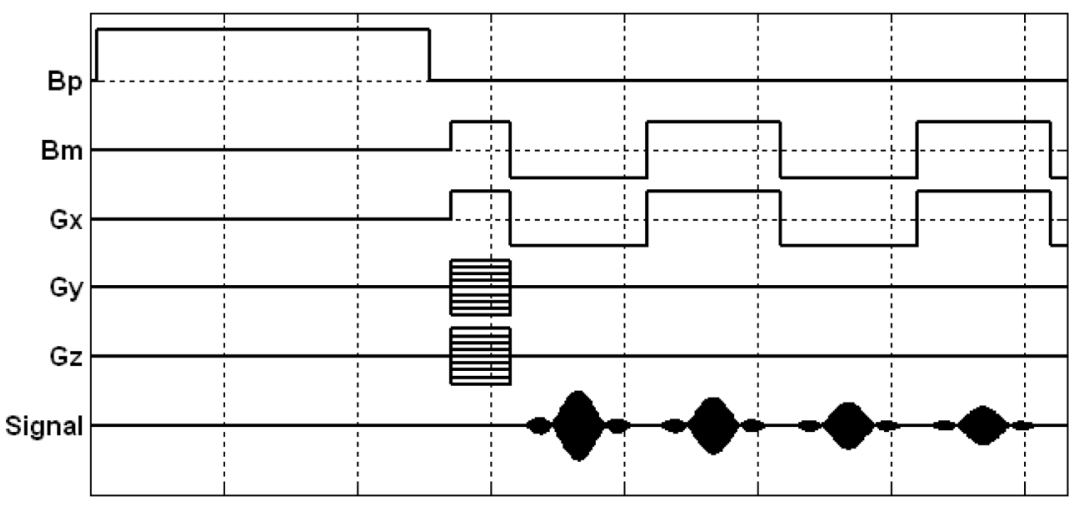

Fig. 1.

3D Fourier multiple gradient echo imaging sequence 


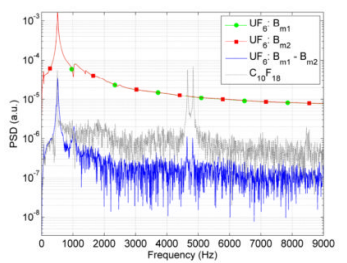

Fig. 2.

Power spectral density of the FIDs from $\mathrm{UF}_{6}$ and the reference liquid fluorine sample perfluorodecalin $\left(\mathrm{C}_{10} \mathrm{~F}_{18}\right)$. The effect of the two-frequency approach is shown as the PSDs from the odd and even epochs have a 100-fold higher noise level than the PSD of the differentiated FIDs. 


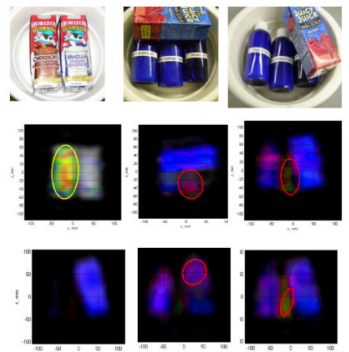

Figure 3.

Top: photographs of items. Middle: 2D images with threat detection. Bottom: 3D slices through the items at top right. 


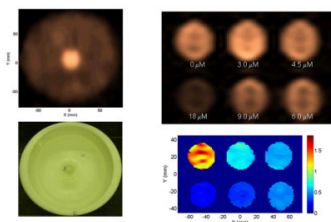

Fig. 4.

Top left MRI of a bottle of Feridex contrast solution $(1.6 \mu \mathrm{M})$ placed in a bowl of water, as shown in the lower left panel. Top right panel MRI retrieved in MagViz showing contrast induced by various concentrations of $20 \mathrm{~nm}$ nanoparticles dissolved in water. Bottom right panel shows $R_{2}$ map of the samples with the color indicating the $R_{2}$ values. 